\title{
New hadron configuration: The double-gluon hybrid state
}

\author{
Hua-Xing Chen $\odot,{ }^{1, *}$ Wei Chen $\oplus^{2, \dagger}$ and Shi-Lin Zhu $\oplus^{3, *}$ \\ ${ }^{1}$ School of Physics, Southeast University, Nanjing 210094, China \\ ${ }^{2}$ School of Physics, Sun Yat-Sen University, Guangzhou 510275, China \\ ${ }^{3}$ School of Physics and Center of High Energy Physics, Peking University, Beijing 100871, China
}

(Received 11 November 2021; accepted 15 February 2022; published 3 March 2022)

\begin{abstract}
This is the first study on the double-gluon hybrid, which consists of one valence quark and one valence antiquark together with two valence gluons. We concentrate on the one with the exotic quantum number $J^{\mathrm{PC}}=2^{+-}$that conventional $\bar{q} q$ mesons cannot reach. We apply the QCD sum rule method to evaluate its mass to be $2.26_{-0.25}^{+0.20} \mathrm{GeV}$, and study its possible decay patterns. Especially, its three-meson decay patterns are generally not suppressed severely compared to two-meson decay patterns, so the $S$-wave three-meson decay channels $f_{1} \omega \pi / f_{1} \rho \pi$ can be useful in identifying its nature, which is of particular importance to the direct test of QCD in the low energy sector.
\end{abstract}

DOI: 10.1103/PhysRevD.105.L051501

\section{INTRODUCTION}

A hybrid state consists of one valence quark and one valence antiquark together with some valence gluons. Its experimental confirmation is a direct test of quantum chromodynamics $(\mathrm{QCD})$ in the low energy sector. Especially, the hybrid states with $J^{\mathrm{PC}}=0^{--} / 0^{+-} / 1^{-+} / 2^{+-} / \cdots$ are of particular interest, since these exotic quantum numbers arise from the manifest gluon degree of freedom and cannot be accessed by conventional $\bar{q} q$ mesons. In the past half century there have been a lot of experimental and theoretical investigations, but their nature remains elusive [1-4].

Up to now there are three candidates observed in experiments with the exotic quantum number $I^{G} J^{\mathrm{PC}}=$ $1^{-} 1^{-+}$, i.e., the $\pi_{1}(1400)$ [5], $\pi_{1}(1600)$ [6,7], and $\pi_{1}(2015)$ [8]. They are possible single-gluon hybrids, which consist of one quark-antiquark pair together with only one valence gluon. Within the flux tube model [9-11] the gluon degree of freedom is modeled as a semiclassical flux tube, and the hybrid with $J^{\mathrm{PC}}=1^{-+}$was found to be around $1.9 \mathrm{GeV}$. The mass calculated using the constituent gluon model is also around $1.9 \mathrm{GeV}$ [12], and those extracted from quenched lattice QCD simulations range from $1.7 \mathrm{GeV}$ to $2.0 \mathrm{GeV}$ [13-19]. We refer to Refs. [20-25] for various studies on them using QCD sum rules and Dyson-Schwinger equation.

\footnotetext{
*hxchen@seu.edu.cn

†chenwei29@mail.sysu.edu.cn

zhusl@pku.edu.cn
}

Published by the American Physical Society under the terms of the Creative Commons Attribution 4.0 International license. Further distribution of this work must maintain attribution to the author(s) and the published article's title, journal citation, and DOI. Funded by SCOAP.
However, the above three $I^{G} J^{\mathrm{PC}}=1^{-} 1^{-+}$structures may also be explained as tetraquark states [26-29]. It is not easy to differentiate the hybrid and tetraquark pictures so far, and this tough problem needs to be solved by experimentalists and theorists together in the future.

In this paper we further investigate the double-gluon hybrid, which consists of one quark-antiquark pair together with two valence gluons. This is motivated by the recent D0 and TOTEM experiments observing the evidence of a $C$-odd three-gluon glueball [30], given that two-gluon glueballs have been studied in detail but are still difficult to be identified unambiguously [1-4]. We construct twelve double-gluon hybrid currents and use them to perform QCD sum rule analyses. As the first study, we concentrate on the one with the exotic quantum number $J^{\mathrm{PC}}=2^{+-}$, which makes it doubly interesting. Among the twelve currents, we find it to be the only one with the mass predicted to be smaller than $3.0 \mathrm{GeV}$, that is $M_{2^{+-}}=$ $2.26_{-0.25}^{+0.20} \mathrm{GeV}$. This mass value is accessible in the BESIII, GlueX, LHC, and PANDA experiments.

We study possible decay patterns of the doublegluon hybrids with $I^{G} J^{\mathrm{PC}}=1^{+} 2^{+-}$and $0^{-} 2^{+-}$, separately for two- and three-meson final states. We propose to search for the one of $I^{G} J^{\mathrm{PC}}=1^{+} 2^{+-}$in its decay channels $\rho f_{0}(980) / \omega \pi / K^{*} \bar{K} / f_{1} \omega \pi / \rho \pi \pi / \cdots$, and the one of $I^{G} J^{\mathrm{PC}}=0^{-} 2^{+-}$in its decay channels $\rho a_{0}(980) / \rho \pi /$ $K^{*} \bar{K} / f_{1} \rho \pi / \omega \pi \pi / \cdots$, both of which are worthy to be searched for in the decay process $J / \psi \rightarrow \pi / \pi \pi / \eta+$ $X\left(\rightarrow K^{*} \bar{K}^{*} / K^{*} \bar{K} \pi / \rho K \bar{K} \rightarrow K \bar{K} \pi \pi\right)$. Especially, their three-meson decay patterns are generally not suppressed severely compared to two-meson decay patterns, since they are both at the $\mathcal{O}\left(\alpha_{s}\right)$ order. Accordingly, the $S$-wave three-meson decay channels $f_{1} \omega \pi / f_{1} \rho \pi$ can be useful in 
distinguishing their nature from the tetraquark picture, therefore, of particular importance to the direct test of QCD in the low energy sector.

\section{DOUBLE-GLUON HYBRID CURRENTS}

As the first step, we use the light up/down quark field $q_{a}(x)$ and the gluon field strength tensor $G_{\mu \nu}^{n}(x)$ to construct double-gluon hybrid currents. Here $a=1 \ldots 3$ and $n=1 \ldots 8$ are color indices; $\mu$ and $\nu$ are Lorentz indices. Besides, we need the antiquark field $\bar{q}_{a}(x)$ and the dual gluon field strength tensor $\tilde{G}_{\mu \nu}^{n}=G^{n, \rho \sigma} \times \epsilon_{\mu \nu \rho \sigma} / 2$.

Generally speaking, one can construct many doublegluon hybrid currents by combining the color-octet quarkantiquark fields,

$$
\begin{array}{ll}
\bar{q}_{a} \lambda_{n}^{a b} q_{b}, & \bar{q}_{a} \lambda_{n}^{a b} \gamma_{5} q_{b}, \\
\bar{q}_{a} \lambda_{n}^{a b} \gamma_{\mu} q_{b}, & \bar{q}_{a} \lambda_{n}^{a b} \gamma_{\mu} \gamma_{5} q_{b}, \quad \bar{q}_{a} \lambda_{n}^{a b} \sigma_{\mu \nu} q_{b},
\end{array}
$$

and the relativistic color-octet double-gluon fields,

$$
d^{n p q} G_{p}^{\alpha \beta} G_{q}^{\gamma \delta}, \quad f^{n p q} G_{p}^{\alpha \beta} G_{q}^{\gamma \delta},
$$

with suitable Lorentz matrices $\Gamma^{\mu \nu \cdots \alpha \beta \gamma \delta}$. Here $d^{n p q}$ and $f^{n p q}$ are totally symmetric and antisymmetric $S U(3)$ structure constants, respectively.

As the first study on the double-gluon hybrid, in the present study we shall investigate the following doublegluon hybrid currents:

$$
\begin{gathered}
J_{0^{-+}}=\bar{q}_{a} \gamma_{5} \lambda_{n}^{a b} q_{b} d^{n p q} g_{s}^{2} G_{p}^{\mu \nu} G_{q, \mu \nu}, \\
J_{0^{--}}=\bar{q}_{a} \gamma_{5} \lambda_{n}^{a b} q_{b} f^{n p q} g_{s}^{2} G_{p}^{\mu \nu} G_{q, \mu \nu}, \\
J_{0^{++}}=\bar{q}_{a} \gamma_{5} \lambda_{n}^{a b} q_{b} d^{n p q} g_{s}^{2} G_{p}^{\mu \nu} \tilde{G}_{q, \mu \nu}, \\
J_{0^{+-}}=\bar{q}_{a} \gamma_{5} \lambda_{n}^{a b} q_{b} f^{n p q} g_{s}^{2} G_{p}^{\mu \nu} \tilde{G}_{q, \mu \nu}, \\
J_{1^{-+}}^{\alpha \beta}=\bar{q}_{a} \gamma_{5} \lambda_{n}^{a b} q_{b} d^{n p q} g_{s}^{2} G_{p}^{\alpha \mu} G_{q, \mu}^{\beta}-\{\alpha \leftrightarrow \beta\}, \\
J_{1^{--}}^{\alpha \beta}=\bar{q}_{a} \gamma_{5} \lambda_{n}^{a b} q_{b} f^{n p q} g_{s}^{2} G_{p}^{\alpha \mu} G_{q, \mu}^{\beta}-\{\alpha \leftrightarrow \beta\}, \\
J_{1^{++}}^{\alpha \beta}=\bar{q}_{a} \gamma_{5} \lambda_{n}^{a b} q_{b} d^{n p q} g_{s}^{2} G_{p}^{\alpha \mu} \tilde{G}_{q, \mu}^{\beta}-\{\alpha \leftrightarrow \beta\}, \\
J_{1^{+-}}^{\alpha \beta}=\bar{q}_{a} \gamma_{5} \lambda_{n}^{a b} q_{b} f^{n p q} g_{s}^{2} G_{p}^{\alpha \mu} \tilde{G}_{q, \mu}^{\beta}-\{\alpha \leftrightarrow \beta\}, \\
J_{2^{-+}}^{\alpha_{1} \beta_{1}, \alpha_{2} \beta_{2}}=\bar{q}_{a} \gamma_{5} \lambda_{n}^{a b} q_{b} d^{n p q} \mathcal{S}\left[g_{s}^{2} G_{p}^{\alpha_{1} \beta_{1}} G_{q}^{\alpha_{2} \beta_{2}}\right], \\
J_{2^{--}}^{\alpha_{1} \beta_{1}, \alpha_{2} \beta_{2}}=\bar{q}_{a} \gamma_{5} \lambda_{n}^{a b} q_{b} f^{n p q} \mathcal{S}\left[g_{s}^{2} G_{p}^{\alpha_{1} \beta_{1}} G_{q}^{\alpha_{2} \beta_{2}}\right], \\
J_{2^{++}}^{\alpha_{1} \beta_{1}, \alpha_{2} \beta_{2}}=\bar{q}_{a} \gamma_{5} \lambda_{n}^{a b} q_{b} d^{n p q} \mathcal{S}\left[g_{s}^{2} G_{p}^{\alpha_{1} \beta_{1}} \tilde{G}_{q}^{\alpha_{2} \beta_{2}}\right], \\
J_{2^{+-}}^{\alpha_{1} \beta_{1}, \alpha_{2} \beta_{2}}=\bar{q}_{a} \gamma_{5} \lambda_{n}^{a b} q_{b} f^{n p q} \mathcal{S}\left[g_{s}^{2} G_{p}^{\alpha_{1} \beta_{1}} \tilde{G}_{q}^{\alpha_{2} \beta_{2}}\right],
\end{gathered}
$$

where $\mathcal{S}$ denotes symmetrization and subtracting trace terms in the two sets $\left\{\alpha_{1} \alpha_{2}\right\}$ and $\left\{\beta_{1} \beta_{2}\right\}$ simultaneously.

The above double-gluon hybrid currents have very clear Lorentz structures, simply because the color-octet quarkantiquark field $\bar{q}_{a} \gamma_{5} \lambda_{n}^{a b} q_{b}$ does not contain any surplus Lorentz index. Besides, this quark-antiquark pair has the $S$-wave spin-parity quantum number $J^{P}=0^{-}$, so these currents are capable of coupling to the lowest-lying doublegluon hybrid states.

\section{QCD SUM RULE ANALYSES}

The method of QCD sum rules has been widely applied in the study of hadron phenomenology [31,32], and in this paper we apply it to study the double-gluon hybrid currents defined in Eqs. (3)-(14). We find that only the doublegluon hybrid state coupled by the current $J_{2^{+-}}^{\alpha_{1} \beta_{1}, \alpha_{2} \beta_{2}}$ has the mass smaller than $3.0 \mathrm{GeV}$. This state has the exotic quantum number $J^{\mathrm{PC}}=2^{+-}$that conventional $\bar{q} q$ mesons cannot reach, making it doubly interesting. Moreover, the current $J_{2^{+-}}^{\alpha_{1} \beta_{1}, \alpha_{2} \beta_{2}}$ contains the double-gluon field with the symmetric spin $J=2$ that cannot (easily) transform to the single-gluon field, making this current significantly different from the single-gluon hybrid current.

We briefly introduce how we use the method of QCD sum rules to study the current $J_{2^{+-}}^{\alpha_{1} \beta_{1}, \alpha_{2} \beta_{2}}$ defined in Eq. (14). Its two-point correlation function

$$
\begin{aligned}
& \Pi^{\alpha_{1} \beta_{1}, \alpha_{2} \beta_{2} ; \alpha_{1}^{\prime} \beta_{1}^{\prime}, \alpha_{2}^{\prime} \beta_{2}^{\prime}}\left(q^{2}\right) \\
& \quad \equiv i \int d^{4} x e^{i q x}\left\langle 0\left|\mathbf{T}\left[J_{2^{+-}}^{\alpha_{1} \beta_{1}, \alpha_{2} \beta_{2}}(x) J_{2^{+-}}^{\alpha_{1}^{\prime} \beta_{1}^{\prime}, \alpha_{2}^{\prime} \beta_{2}^{\prime} \dagger}(0)\right]\right| 0\right\rangle \\
& \quad=\mathcal{S}^{\prime}\left[g^{\alpha_{1} \alpha_{1}^{\prime}} g^{\beta_{1} \beta_{1}^{\prime}} g^{\alpha_{2} \alpha_{2}^{\prime}} g^{\beta_{2} \beta_{2}^{\prime}}\right] \Pi\left(q^{2}\right),
\end{aligned}
$$

can be investigated at both hadron and quark-gluon levels. Here $\mathcal{S}^{\prime}$ denotes antisymmetrization in the four sets $\left\{\alpha_{1} \beta_{1}\right\},\left\{\alpha_{2} \beta_{2}\right\},\left\{\alpha_{1}^{\prime} \beta_{1}^{\prime}\right\}$, and $\left\{\alpha_{2}^{\prime} \beta_{2}^{\prime}\right\}$, and symmetrization and subtracting trace terms in the four sets $\left\{\alpha_{1} \alpha_{2}\right\},\left\{\beta_{1} \beta_{2}\right\}$, $\left\{\alpha_{1}^{\prime} \alpha_{2}^{\prime}\right\}$, and $\left\{\beta_{1}^{\prime} \beta_{2}^{\prime}\right\}$, simultaneously.

The spectral density $\rho(s) \equiv \operatorname{Im} \Pi(s) / \pi$ can be extracted from Eq. (15) through the dispersion relation

$$
\Pi\left(q^{2}\right)=\int_{0}^{\infty} \frac{\rho(s)}{s-q^{2}-i \varepsilon} d s .
$$

At the hadron level we parametrize it using one pole dominance for the possible ground state $\left|X ; 2^{+-}\right\rangle$together with the continuum contribution:

$$
\begin{aligned}
\rho_{\text {phen }}(s) & \equiv \sum_{n} \delta\left(s-M_{n}^{2}\right)\langle 0|J| n\rangle\left\langle n\left|J^{\dagger}\right| 0\right\rangle \\
& =f_{X}^{2} \delta\left(s-M_{X}^{2}\right)+\text { continuum. }
\end{aligned}
$$

At the quark-gluon level we calculate Eq. (15) and extract $\rho_{\mathrm{OPE}}(s)$ using the method of operator product expansion 
(OPE). After performing the Borel transformation to Eq. (16) at both hadron and quark-gluon levels, we obtain

$\Pi\left(s_{0}, M_{B}^{2}\right) \equiv f_{X}^{2} e^{-M_{X}^{2} / M_{B}^{2}}=\int_{0}^{s_{0}} \rho_{\mathrm{OPE}}(s) e^{-s / M_{B}^{2}} d s$,

where we have approximated the continuum using the OPE spectral density above the threshold value $s_{0}$.

Finally, we calculate the mass of $\left|X ; 2^{+-}\right\rangle$through

$$
M_{X}^{2}\left(s_{0}, M_{B}\right)=\frac{\int_{0}^{s_{0}} s \rho_{\mathrm{OPE}}(s) e^{-s / M_{B}^{2}} d s}{\int_{0}^{s_{0}} \rho_{\mathrm{OPE}}(s) e^{-s / M_{B}^{2}} d s} .
$$

In the present study we take into account the Feynman diagrams depicted in Fig. 1, and calculate $\rho_{\mathrm{OPE}}(s)$ up to the

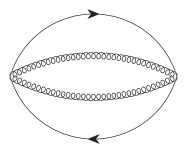

(a)

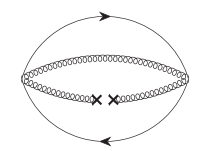

(b-1)

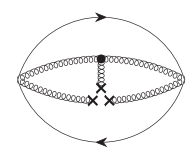

(c-1)

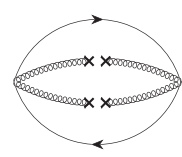

(b-2)

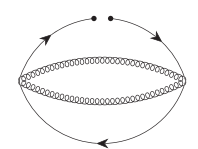

(b-3)

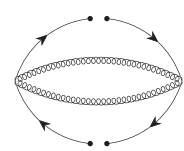

$(\mathrm{b}-4)$

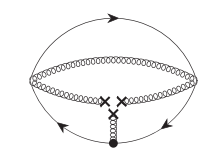

(c-2)

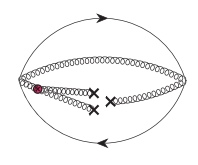

(c-3)

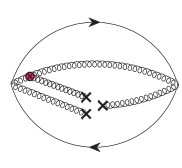

(c-4)

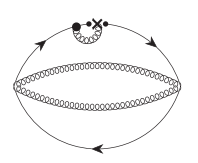

(d-1)

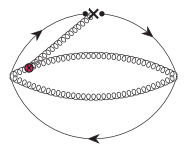

(d-4)

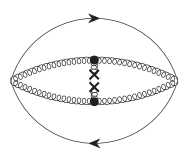

$(\mathrm{e}-1)$

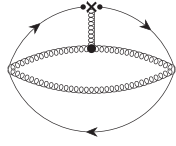

$(\mathrm{d}-2)$

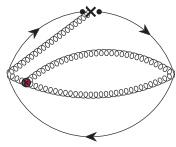

$(d-5)$

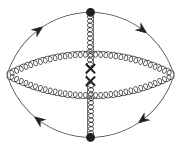

(e-2)

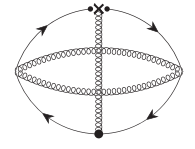

(d-3)

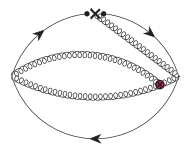

$(d-6)$

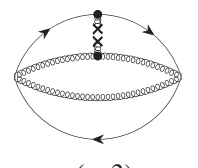

$(\mathrm{e}-3)$
FIG. 1. Feynman diagrams for the double-gluon hybrid, including the perturbative term, the quark condensate $\langle\bar{q} q\rangle$, the quark-gluon mixed condensate $\left\langle\bar{g}_{s} q \sigma G q\right\rangle$, the two-gluon condensate $\left\langle g_{s}^{2} G G\right\rangle$, the three-gluon condensate $\left\langle g_{s}^{3} G^{3}\right\rangle$, and their combinations. The diagrams (a) and (b-i) are proportional to $\alpha_{s}^{2} \times g_{s}^{0}$, the diagrams (c-i) and (d-i) are proportional to $\alpha_{s}^{2} \times g_{s}^{1}$, and the diagrams (e-i) are proportional to $\alpha_{s}^{2} \times g_{s}^{2}$. dimension eight $(D=8)$ condensates. The gluon field strength tensor $G_{\mu \nu}^{n}$ is defined as

$$
G_{\mu \nu}^{n}=\partial_{\mu} A_{\nu}^{n}-\partial_{\nu} A_{\mu}^{n}+g_{s} f^{n p q} A_{p, \mu} A_{q, \nu},
$$

so it can be naturally separated into two parts. We depict the former two terms using the single-gluon-line, and the third term using the double-gluon-line with a red vertex, e.g., the diagram depicted in Fig. 1(c-3).

We calculate the spectral density from the current $J_{2^{+-}}^{\alpha_{1} \beta_{1}, \alpha_{2} \beta_{2}}$ to be

$$
\begin{aligned}
\rho_{\mathrm{OPE}}(s)= & \frac{\alpha_{s}^{2} s^{5}}{80640 \pi^{4}}+\left(\frac{\alpha_{s}\left\langle g_{s}^{2} G G\right\rangle}{3840 \pi^{3}}+\frac{7 \alpha_{s}^{2}\left\langle g_{s}^{2} G G\right\rangle}{61440 \pi^{4}}\right) s^{3} \\
& +\left(\frac{\alpha_{s}^{2}\langle\bar{q} q\rangle^{2}}{9}-\frac{\alpha_{s}\left\langle g_{s}^{3} G^{3}\right\rangle}{1536 \pi^{3}}\right) s^{2} \\
& +\left(-\frac{2 \alpha_{s}^{2}\langle\bar{q} q\rangle\left\langle\bar{g}_{s} q \sigma G q\right\rangle}{9}-\frac{\alpha_{s}\left\langle g_{s}^{2} G G\right\rangle^{2}}{18432 \pi^{3}}\right) s,
\end{aligned}
$$

where we have taken into account all the diagrams proportional to $\alpha_{s}^{2} \times g_{s}^{0}$ and $\alpha_{s}^{2} \times g_{s}^{1}$; while there are so many diagrams proportional to $\alpha_{s}^{2} \times g_{s}^{2}$, and we have kept only three of them, as depicted in Fig. 1(e-i).

\section{NUMERICAL ANALYSES}

We study the sum rules given in Eq. (21) numerically using the following values for various QCD parameters at the renormalization scale $2 \mathrm{GeV}$ and the QCD scale $\Lambda_{\mathrm{QCD}}=300 \mathrm{MeV}$ [1,33-37]:

$$
\begin{aligned}
\alpha_{s}\left(Q^{2}\right) & =\frac{4 \pi}{11 \ln \left(Q^{2} / \Lambda_{\mathrm{QCD}}^{2}\right)}, \\
\langle\bar{q} q\rangle & =-(0.240 \pm 0.010)^{3} \mathrm{GeV}^{3}, \\
\left\langle g_{s} \bar{q} \sigma G q\right\rangle & =(0.8 \pm 0.2) \times\langle\bar{q} q\rangle \mathrm{GeV}^{2}, \\
\left\langle\alpha_{s} G G\right\rangle & =(6.35 \pm 0.35) \times 10^{-2} \mathrm{GeV}^{4}, \\
\left\langle g_{s}^{3} G^{3}\right\rangle & =(8.2 \pm 1.0) \times\left\langle\alpha_{s} G G\right\rangle \mathrm{GeV}^{2} .
\end{aligned}
$$

As shown in Eq. (19), the mass of $\left|X ; 2^{+-}\right\rangle$depends on the Borel mass $M_{B}$ and the threshold value $s_{0}$. To insure the convergence of Eq. (21), we require a) the $\alpha_{s}^{2} \times g_{s}^{2}$ terms to be less than $5 \%$, and b) the $D=8$ terms to be less than $10 \%$ :

$$
\begin{gathered}
\mathrm{CVG} \equiv\left|\frac{\Pi^{g_{s}^{n=6}}\left(s_{0}, M_{B}^{2}\right)}{\Pi\left(s_{0}, M_{B}^{2}\right)}\right| \leq 5 \%, \\
\mathrm{CVG}^{\prime} \equiv\left|\frac{\Pi^{\mathrm{D}=8}\left(s_{0}, M_{B}^{2}\right)}{\Pi\left(s_{0}, M_{B}^{2}\right)}\right| \leq 10 \%
\end{gathered}
$$

To insure the one-pole-dominance assumption, we require the pole contribution (PC) to be larger than $40 \%$ : 


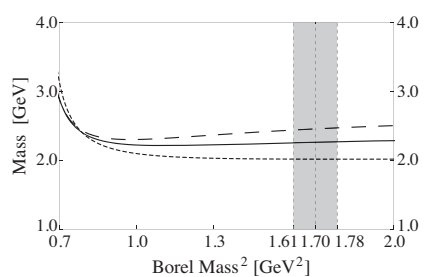

(a)

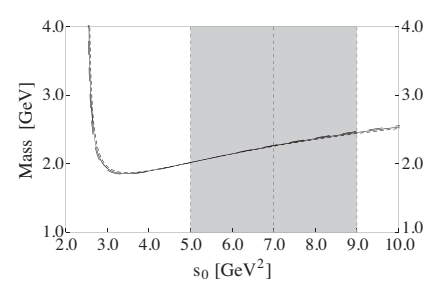

(b)
FIG. 2. Mass of the double-gluon hybrid $\left|X ; 2^{+-}\right\rangle$as a function of the Borel mass $M_{B}$ (a) and the threshold value $s_{0}$ (b). In the subfigure (a) the short-dashed/solid/long-dashed curves are obtained by setting $s_{0}=5.0 / 7.0 / 9.0 \mathrm{GeV}^{2}$, respectively. In the subfigure (b) the short-dashed/solid/long-dashed curves are obtained by setting $M_{B}^{2}=1.61 / 1.70 / 1.78 \mathrm{GeV}^{2}$, respectively.

$$
\mathrm{PC} \equiv\left|\frac{\Pi\left(s_{0}, M_{B}^{2}\right)}{\Pi\left(\infty, M_{B}^{2}\right)}\right| \geq 40 \%
$$

Altogether we determine the Borel window to be $1.61 \mathrm{GeV}^{2} \leq M_{B}^{2} \leq 1.78 \mathrm{GeV}^{2}$ when setting $s_{0}=7.0 \mathrm{GeV}^{2}$.

We redo the same procedures by changing $s_{0}$, and find that there are nonvanishing Borel windows as long as $s_{0} \geq s_{0}^{\min }=6.3 \mathrm{GeV}^{2}$. Accordingly, we set $s_{0}$ to be about $10 \%$ larger, and determine our working regions to be $5.0 \mathrm{GeV}^{2} \leq s_{0} \leq 9.0 \mathrm{GeV}^{2}$ and $1.61 \mathrm{GeV}^{2} \leq M_{B}^{2} \leq$ $1.78 \mathrm{GeV}^{2}$. The mass of $\left|X ; 2^{+-}\right\rangle$is evaluated to be

$$
\begin{aligned}
M_{\left|X ; 2^{+-}\right\rangle} & =2.26_{-0.25}^{+0.19} \pm 0.07 \pm 0.03 \mathrm{GeV} \\
& =2.26_{-0.25}^{+0.20} \mathrm{GeV}
\end{aligned}
$$

whose uncertainty is due to the threshold value $s_{0}$, Borel mass $M_{B}$, and various quark and gluon parameters listed in Eqs. (22), respectively. We show it in Fig. 2 as a function of the Borel mass $M_{B}$ and the threshold value $s_{0}$. This mass value is obtained for both isoscalar and isovector states, so actually we cannot differentiate them in the present QCD sum rule study.

For completeness, we also use the other eleven hybrid currents defined in Eqs. (3)-(13) to perform QCD sum rule analyses. We explicitly prove the four currents $J_{0^{--} / 0^{+-} / 1^{-+} / 1^{++}}^{\cdots}$ to be zero, while masses extracted from the seven currents $J_{0^{-+}}^{\ldots} / 0^{++} / 1^{--} / 1^{+-} / 2^{--} / 2^{-+} / 2^{++}$are all larger than $3.0 \mathrm{GeV}$. We leave their detailed discussions for our future studies.

\section{DECAY ANALYSES}

The double-gluon hybrid can decay after exciting two $\bar{q} q / \bar{s} s(q=u / d)$ pairs from two gluons, followed by recombining three color-octet $\bar{q} q / \bar{s} s$ pairs into two color-singlet mesons or three mesons, as depicted in Fig. 3. These two possible decay processes are both at the $\mathcal{O}\left(\alpha_{s}\right)$ order, so three-meson decay patterns are generally not suppressed severely compared to two-meson

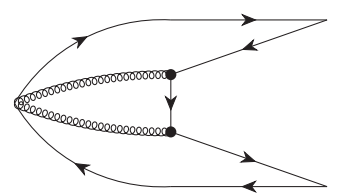

(a)

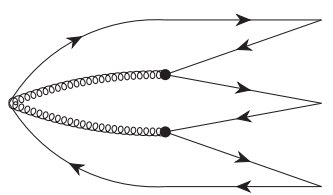

(b)
FIG. 3. Possible decay processes of the double-gluon hybrid.

decay patterns, or even enhanced due to the quark-antiquark annihilation during the two-meson decay process. This behavior can be useful in identifying the nature of the double-gluon hybrid.

To investigate decay properties of the double-gluon hybrid, we assume its final quark content to be either

$$
(\bar{q} q)_{\mathbf{8}_{C}} \times(\bar{q} q+\bar{s} s)_{\mathbf{8}_{C}} \rightarrow(\bar{q} q)_{\mathbf{1}_{C}}(\bar{q} q)_{\mathbf{1}_{C}}+(\bar{q} s)_{\mathbf{1}_{C}}(\bar{s} q)_{\mathbf{1}_{C}},
$$

or

$$
\begin{aligned}
(\bar{q} q)_{\mathbf{8}_{C}} \times(\bar{q} q+\bar{s} s)_{\mathbf{8}_{C}}^{2} \rightarrow & (\bar{q} q)_{\mathbf{1}_{C}}(\bar{q} q)_{\mathbf{1}_{C}}(\bar{q} q)_{\mathbf{1}_{C}} \\
& +(\bar{q} q)_{\mathbf{1}_{C}}(\bar{q} s)_{\mathbf{1}_{C}}(\bar{s} q)_{\mathbf{1}_{C}} \\
& +(\bar{s} s)_{\mathbf{1}_{C}}(\bar{q} s)_{\mathbf{1}_{C}}(\bar{s} q)_{\mathbf{1}_{C}} .
\end{aligned}
$$

Accordingly, we list some possible decay patterns of the double-gluon hybrids with the exotic quantum numbers $I^{G} J^{\mathrm{PC}}=1^{+} 2^{+-}$and $0^{-} 2^{+-}$in Table I, separately for twoand three-meson decay processes. The one of $I^{G} J^{\mathrm{PC}}=$ $1^{+} 2^{+-}$may be observed in its two-meson decay channels $\rho f_{0}(980) / \omega \pi / K^{*} \bar{K} / \cdots$ and three-meson decay channels $f_{1} \omega \pi / \rho \pi \pi / \cdots$; the one of $I^{G} J^{\mathrm{PC}}=0^{-} 2^{+-}$may be observed in its two-meson decay channels $\rho a_{0}(980) / \rho \pi /$ $K^{*} \bar{K} / \cdots$ and three-meson decay channels $f_{1} \rho \pi / \omega \pi \pi / \cdots$. Especially, both of them are worthy to be searched for in the decay process $J / \psi \rightarrow \pi / \pi \pi / \eta+X\left(\rightarrow K^{*} \bar{K}^{*} / K^{*} \bar{K} \pi /\right.$ $\rho K \bar{K} \rightarrow K \bar{K} \pi \pi)$, and the $S$-wave three-meson decay channels $f_{1} \omega \pi / f_{1} \rho \pi$ can be useful in identifying their nature.

TABLE I. Some possible two- and three-meson decay patterns of the double-gluon hybrids with the exotic quantum numbers $I^{G} J^{\mathrm{PC}}=1^{+} 2^{+-}$and $0^{-} 2^{+-}$.

\begin{tabular}{lcc}
\hline \hline Two-Meson & $1^{+} 2^{+-}$ & $0^{-} 2^{+-}$ \\
\hline S-wave & $K_{2}^{*} \bar{K}_{0}^{*}$ & \\
P-wave & $h_{1} \pi, a_{1} \pi, a_{2} \pi, b_{1} \eta, \rho f_{0}$ & $b_{1} \pi, h_{1} \eta, \rho a_{0}$ \\
& $K_{1} \bar{K}, K_{2}^{*} \bar{K}, K^{*} \bar{K}_{0}^{*}$ & \\
D-wave & $\rho^{+} \rho^{-}, \omega \pi, \rho \eta, \rho \eta^{\prime}$ & $\rho \pi, \omega \eta, \omega \eta^{\prime}$ \\
& $K^{*} \bar{K}, K^{*} \bar{K}^{*}$ & $0^{-} 2^{+-}$ \\
Three-Meson & $1^{+} 2^{+-}$ & $f_{1} \rho \pi, a_{1} \omega \pi$ \\
S-wave & $f_{1} \omega \pi, a_{1} \rho \pi$ & $\omega \pi \pi, \rho \eta \pi$ \\
P-wave & $\rho \pi \pi, \omega \eta \pi, \rho \eta \eta$ & \multicolumn{2}{c}{$K^{*} \bar{K} \pi, \rho K \bar{K}, \omega K \bar{K}$} \\
\hline \hline
\end{tabular}




\section{SUMMARY}

As the first study on the double-gluon hybrid, we systematically construct twelve double-gluon hybrid currents and use them to perform QCD sum rule analyses. These currents are constructed by using the $S$-wave color-octet quark-antiquark field $\bar{q}_{a} \gamma_{5} \lambda_{n}^{a b} q_{b}$ together with the relativistic color-octet double-gluon fields. Among them, we find that only the double-gluon hybrid state coupled by the current $J_{2^{+-}}^{\alpha_{1} \beta_{1}, \alpha_{2} \beta_{2}}$ has the mass smaller than $3.0 \mathrm{GeV}$, that is

$$
M_{\left|X ; 2^{+-}\right\rangle}=2.26_{-0.25}^{+0.20} \mathrm{GeV},
$$

which is accessible in the BESIII, GlueX, LHC, and PANDA experiments. Moreover, this state has the exotic quantum number $J^{\mathrm{PC}}=2^{+-}$that conventional $\bar{q} q$ mesons cannot reach, making it doubly interesting.

We study its possible decay patterns separately for twoand three-meson final states. We propose to search for the one of $I^{G} J^{\mathrm{PC}}=1^{+} 2^{+-}$in its decay channels $\rho f_{0}(980) /$ $\omega \pi / K^{*} \bar{K} / f_{1} \omega \pi / \rho \pi \pi / \cdots$, and the one of $I^{G} J^{\mathrm{PC}}=0^{-} 2^{+-}$ in its decay channels $\rho a_{0}(980) / \rho \pi / K^{*} \bar{K} / f_{1} \rho \pi / \omega \pi \pi / \cdots$, both of which are worthy to be searched for in the decay process $J / \psi \rightarrow \pi / \pi \pi / \eta+X\left(\rightarrow K^{*} \bar{K}^{*} / K^{*} \bar{K} \pi / \rho K \bar{K} \rightarrow\right.$ $K \bar{K} \pi \pi)$. Especially, their three-meson decay patterns are generally not suppressed severely compared to two-meson decay patterns, since they are both at the $\mathcal{O}\left(\alpha_{s}\right)$ order. Accordingly, the $S$-wave three-meson decay channels $f_{1} \omega \pi / f_{1} \rho \pi$ can be useful in identifying their nature, therefore, are of particular importance to the direct test of QCD in the low energy sector.

\section{ACKNOWLEDGMENTS}

This project is supported by the National Natural Science Foundation of China under Grants No. 11975033, No. 12075019, No. 12175318, and No. 12070131001, the National Key R\&D Program of China under Contracts No. 2020YFA0406400, the Jiangsu Provincial DoubleInnovation Program under Grant No. JSSCRC2021488, and the Fundamental Research Funds for the Central Universities.
[1] P. A. Zyla et al. (Particle Data Group), Review of particle physics, Prog. Theor. Exp. Phys. 2020, 083C01 (2020).

[2] E. Klempt and A. Zaitsev, Glueballs, hybrids, multiquarks: Experimental facts versus QCD inspired concepts, Phys. Rep. 454, 1 (2007).

[3] C. A. Meyer and E. S. Swanson, Hybrid mesons, Prog. Part. Nucl. Phys. 82, 21 (2015).

[4] H. X. Chen, W. Chen, X. Liu, and S. L. Zhu, The hiddencharm pentaquark and tetraquark states, Phys. Rep. 639, 1 (2016).

[5] D. Alde et al. (LAPP Collaboration), Evidence for a $1^{-+}$ exotic meson, Phys. Lett. B 205, 397 (1988).

[6] E. I. Ivanov et al. (E852 Collaboration), Observation of Exotic Meson Production in the Reaction $\pi^{-} p \rightarrow \eta^{\prime} \pi^{-} p$ at $18 \mathrm{GeV} / c$, Phys. Rev. Lett. 86, 3977 (2001).

[7] M. Alekseev et al. (COMPASS Collaboration), Observation of a $J^{\mathrm{PC}}=1^{-+}$Exotic Resonance in Diffractive Dissociation of $190 \mathrm{GeV} / c \pi^{-}$into $\pi^{-} \pi^{-} \pi^{+}$, Phys. Rev. Lett. 104, 241803 (2010).

[8] J. Kuhn et al. (E852 Collaboration), Exotic meson production in the $f_{1}(1285) \pi^{-}$system observed in the reaction $\pi^{-} p \rightarrow \eta \pi^{+} \pi^{-} \pi^{-} p$ at $18 \mathrm{GeV} / c$, Phys. Lett. B 595, 109 (2004).

[9] N. Isgur and J. E. Paton, Flux-tube model for hadrons in QCD, Phys. Rev. D 31, 2910 (1985).

[10] P. R. Page, E. S. Swanson, and A. P. Szczepaniak, Hybrid meson decay phenomenology, Phys. Rev. D 59, 034016 (1999).

[11] T. Burns and F. E. Close, Hybrid-meson properties in lattice QCD and flux-tube models, Phys. Rev. D 74, 034003 (2006).
[12] F. Iddir and L. Semlala, Hybrid states from constituent glue model, Int. J. Mod. Phys. A 23, 5229 (2008).

[13] C. McNeile, C. W. Bernard, T. A. DeGrand, C. E. DeTar, S. A. Gottlieb, U. M. Heller, J. Hetrick, R. Sugar, and D. Toussaint, Exotic meson spectroscopy from the clover action at $\beta=5.85$ and 6.15, Nucl. Phys. B Proc. Suppl. 73, 264 (1999).

[14] P. Lacock et al. (TXL Collaboration), Hybrid and orbitally excited mesons in full QCD, Nucl. Phys. B Proc. Suppl. 73, 261 (1999).

[15] C. Bernard, T. Burch, E. B. Gregory, D. Toussaint, C. E. DeTar, J. Osborn, S. A. Gottlieb, U. M. Heller, and R. Sugar, Lattice calculation of $1^{-+}$hybrid mesons with improved Kogut-Susskind fermions, Phys. Rev. D 68, 074505 (2003).

[16] J. N. Hedditch, W. Kamleh, B. G. Lasscock, D. B. Leinweber, A. G. Williams, and J. M. Zanotti, $1^{-+}$exotic meson at light quark masses, Phys. Rev. D 72, 114507 (2005).

[17] J. J. Dudek, R. G. Edwards, M. J. Peardon, D. G. Richards, and C.E. Thomas, Highly Excited and Exotic Meson Spectrum from Dynamical Lattice QCD, Phys. Rev. Lett. 103, 262001 (2009).

[18] J. J. Dudek, R. G. Edwards, M. J. Peardon, D. G. Richards, and C. E. Thomas, Toward the excited meson spectrum of dynamical QCD, Phys. Rev. D 82, 034508 (2010).

[19] R. A. Briceno, J. J. Dudek, and R. D. Young, Scattering processes and resonances from lattice QCD, Rev. Mod. Phys. 90, 025001 (2018).

[20] J. Govaerts, F. de Viron, D. Gusbin, and J. Weyers, QCD sum rules and hybrid mesons, Nucl. Phys. B248, 1 (1984).

[21] K. G. Chetyrkin and S. Narison, Light hybrid mesons in QCD, Phys. Lett. B 485, 145 (2000). 
[22] H. Y. Jin, J. G. Körner, and T. G. Steele, Improved determination of the mass of the $1^{-+}$light hybrid meson from QCD sum rules, Phys. Rev. D 67, 014025 (2003).

[23] H. X. Chen, Z. X. Cai, P. Z. Huang, and S. L. Zhu, Decay properties of the $1^{-+}$hybrid state, Phys. Rev. D 83, 014006 (2011).

[24] Z. R. Huang, H. Y. Jin, T. G. Steele, and Z. F. Zhang, Revisiting the $b_{1} \pi$ and $\rho \pi$ decay modes of the $1^{-+}$light hybrid state with light-cone QCD sum rules, Phys. Rev. D 94, 054037 (2016).

[25] S. S. Xu, Z. F. Cui, L. Chang, J. Papavassiliou, C. D. Roberts, and H.S. Zong, New perspective on hybrid mesons, Eur. Phys. J. A 55, 113 (2019).

[26] S. U. Chung, E. Klempt, and J. G. Körner, SU(3) classification of $p$-wave $\eta \pi$ and $\eta^{\prime} \pi$ systems, Eur. Phys. J. A 15, 539 (2002).

[27] H. X. Chen, A. Hosaka, and S. L. Zhu, $I^{G} J^{\mathrm{PC}}=1^{-} 1^{-+}$ tetraquark states, Phys. Rev. D 78, 054017 (2008).

[28] H. X. Chen, A. Hosaka, and S. L. Zhu, $I^{G} J^{\mathrm{PC}}=0^{+} 1^{-+}$ tetraquark state, Phys. Rev. D 78, 117502 (2008).

[29] S. Narison, $1^{-+}$light exotic mesons in QCD, Phys. Lett. B 675, 319 (2009).

[30] V. M. Abazov et al. (TOTEM and D0 Collaborations), Odderon Exchange from Elastic Scattering Differences between $p p$ and $p \bar{p}$ Data at $1.96 \mathrm{TeV}$ and from pp Forward
Scattering Measurements, Phys. Rev. Lett. 127, 062003 (2021).

[31] M. A. Shifman, A. I. Vainshtein, and V. I. Zakharov, QCD and resonance physics. Theoretical foundations, Nucl. Phys. B147, 385 (1979).

[32] L. J. Reinders, H. Rubinstein, and S. Yazaki, Hadron properties from QCD sum rules, Phys. Rep. 127, 1 (1985).

[33] A. A. Ovchinnikov and A.A. Pivovarov, QCD sum rule calculation of the quark gluon condensate, Yad. Fiz. 48, 1135 (1988) [Sov. J. Nucl. Phys. 48, 721 (1988)].

[34] M. Jamin, Flavour-symmetry breaking of the quark condensate and chiral corrections to the Gell-Mann-OakesRenner relation, Phys. Lett. B 538, 71 (2002).

[35] V. Gimenez, V. Lubicz, F. Mescia, V. Porretti, and J. Reyes, Operator product expansion and quark condensate from lattice QCD in coordinate space, Eur. Phys. J. C 41, 535 (2005).

[36] S. Narison, Gluon condensates and precise $\bar{m}_{c, b}$ from QCDmoments and their ratios to order $\alpha_{s}^{3}$ and $\left\langle G^{4}\right\rangle$, Phys. Lett. B 706, 412 (2012).

[37] S. Narison, QCD parameter correlations from heavy quarkonia, Int. J. Mod. Phys. A 33, 1850045 (2018); 33, 1892004(A) (2018). 